\title{
Ambient Monitoring of Gait and Machine Learning Models for Dynamic and Short-Term Falls Risk Assessment in People With Dementia
}

This paper was downloaded from TechRxiv (https://www.techrxiv.org).

\section{LICENSE}

CC BY 4.0

SUBMISSION DATE / POSTED DATE

$06-11-2021 / 14-11-2021$

\section{CITATION}

Korhani, Navid; Taati, Babak; laboni, Andrea; Sabo, Andrea; Mehdizadeh, Sina; Flint, Alastair; et al. (2021): Ambient Monitoring of Gait and Machine Learning Models for Dynamic and Short-Term Falls Risk Assessment in People With Dementia. TechRxiv. Preprint. https://doi.org/10.36227/techrxiv.16943395.v1

$\mathrm{DOI}$ 


\title{
Ambient Monitoring of Gait and Machine Learning Models for Dynamic and Short-Term Falls Risk Assessment in People With Dementia
}

\author{
Navid Korhani, Andrea Sabo, Sina Mehdizadeh, Avril Mansfield, Alastair Flint, Andrea laboni, Babak Taati
}

\begin{abstract}
Falls are a leading cause of morbidity and mortality in older adults with dementia residing in long-term care. Having access to a frequently updated and accurate estimate of shortterm fall risk for each resident will enable care staff to provide targeted interventions to prevent falls and resulting injuries. To this end, machine learning models to estimate and frequently update the short-term fall risk were trained on longitudinal data from $\mathbf{5 4}$ older adult participants with dementia. Data from each participant included baseline clinical assessments of gait, mobility, and fall risk at the time of admission, daily medication intake in three medication categories, and frequent assessments of gait performed via a computer vision-based ambient monitoring system. Systematic ablations investigated the effects of various hyperparameters and feature sets and experimentally identified differential contributions from baseline clinical assessments, ambient gait analysis, and daily medication intake. In leave-one-subject-out cross-validation, the best performing model detects falls over the next 4 weeks with a sensitivity and specificity of 75.5 and 71.9 , respectively, and achieved an area under the receiver operating characteristic curve (AUROC) of 75.8. By contrast, the best model excluding ambient gait features achieved an AUROC of 70.5 with a sensitivity and specificity of 64.7 and 74.4 , respectively. Future research will focus on externally validating these findings to prepare for the implementation of this technology to reduce fall and fall-related injuries in long-term care.
\end{abstract}

Index Terms_-Fall Risk Assessment, Fall Prediction, Dementia, Gait, Machine Learning, Ambient Monitoring

This work was supported by the Walter and Maria Schroeder Institute for Brain Innovation and Recovery; KITE - Toronto Rehabilitation Institute - University Health Network; the Canadian Institute of Health Research (ClHR), the Natural Sciences and Engineering Research Council; Alzheimer's Association \& Brain Canada; and AMS Healthcare Fellowship in Compassion and Artificial Intelligence.

N. Korhani is with KITE, Toronto Rehabilitation Institute, University Health Network (UHN), Toronto, Ontario, Canada; and the Department of Computer Science, University of Toronto, Toronto, Ontario, Canada. (e-mail: navid.korhani@mail.utoronto.ca)

A. Sabo is with KITE, Toronto Rehabilitation Institute, University Health Network (UHN), Toronto, Ontario, Canada; and the Institute of Biomedical Engineering, University of Toronto, Toronto, Ontario, Canada. (e-mail: Andrea.Sabo@mail.utoronto.ca)

S. Mehdizadeh is with KITE, Toronto Rehabilitation Institute, UHN, Toronto, Ontario, Canada. (e-mail: Sina.Mehdizadeh@uhnresearch.ca)

A. Mansfield is with KITE, Toronto Rehabilitation Institute, University Health Network, Toronto, Ontario, Canada; and Department of Physical Therapy, University of Toronto, Toronto, Ontario, Canada; and the Evaluative Clinical Sciences, Hurvitz Brain Sciences Program, Sunnybrook Research Institute, Toronto, ON, Canada. (e-mail: avril.mansfield@uhn.ca)

A. Flint is with KITE, Toronto Rehabilitation Institute, University Health Network (UHN), Toronto, Ontario, Canada; and the Department of Psychiatry, University of Toronto, Toronto, Ontario, Canada; and the Center for Mental Health, University Health Network, Toronto, Ontario, Canada. (e-mail: alastair.flint@uhn.ca)

A. Iaboni is with KITE, Toronto Rehabilitation Institute, UHN, Toronto, Canada; and the Department of Psychiatry, University of Toronto, Toronto, Ontario, Canada; and the Centre for Mental Health, UHN, Toronto, ON Canada. (e-mail: Andrea.laboni@uhn.ca)

B. Taati is with KITE, Toronto Rehabilitation Institute, UHN, Toronto, Ontario, Canada; the Department of Computer Science, University of Toronto, Toronto, Ontario, Canada; and the Institute of Biomedical Engineering, University of Toronto, Toronto, Ontario, Canada. (email: Babak.Taati@uhn.ca)

\section{INTRODUCTION}

Falls are one of the leading causes of injury, loss of independence, and mortality in older adults [1]. The annual incidence of falls in older adults with dementia is $70 \%-80 \%$ [2], approximately twice the incidence of falls in cognitively healthy older adults [3], [4]. In longterm care (LTC) homes, about $25 \%$ of falls result in injuries, $15 \%$ severe enough to require medical care [5]. Since falls have a major impact on functional ability, caregiver burden, and quality of life, fall prevention has become an important public health goal in high-risk populations such as residents of LTC centers [6].

An important part of a falls prevention plan is determining the level of risk of falling. One of the shortcomings of many existing approaches is that their risk evaluation is solely based on a static baseline assessment of individuals, which identifies the risk of falling over time frames of months to years [7]. By this standard, most LTC residents are accurately categorized as having a high risk of falling, making it difficult for care staff to act upon this information. While it may be useful to be aware of someone's long-term risk of falls, acute changes in gait and dynamic stability may be predictive of falls in the short-term, i.e. days to weeks. For instance, new medications, failing health, or declines in cognition may detectably alter falls risk in the days immediately preceding a fall. To improve upon static assessments and to identify individuals at imminent risk of falling, one needs to incorporate frequent (daily or weekly) measurements that may correlate with or cause falls in the short term, e.g. over the next few days or weeks.

There are many intrinsic and extrinsic factors that contribute to falls [8]. Extrinsic or environmental fall risk factors include slippery surfaces, tripping hazards, dim lighting, etc. Examples of intrinsic fall risk factors include gait and balance problems, poor vision, poor reaction time, and muscle weakness. In the absence of full awareness of all intrinsic and extrinsic factors contributing to falls, it is impossible to fully and accurately predict future falls. It is, however, still very useful to try to build and validate techniques that assess fall risk with moderate accuracy, as LTC care staff can take this information into account in targeted interventions. For example, if a fall risk assessment system could identify LTC residents with a rapid rise in their estimated risk of falling (based on a combination of specific intrinsic and/or extrinsic risk factors), clinicians and care staff can attend to those residents and, for instance, examine their health status and recent changes in medications as possible causes, or implement harm reduction strategies such as hip protectors. Such targeted interventions may improve the efficiency of LTC staff in providing timely care and reducing fall risk.

The objective of this study was to develop a dynamic fall risk assessment tool - i.e. one that updates its risk estimate over time - for older adults with dementia. To this end, we applied a machine learning approach and leave-one-subject-out cross-validation to examine the predictive power of various combination of predictors including static baseline assessments, medication exposures over time, and gait parameters measured via frequent ambient monitoring. Our work shows, for the first time, that fall risk predictions in older adults with dementia can be improved using ambient gait monitoring. 


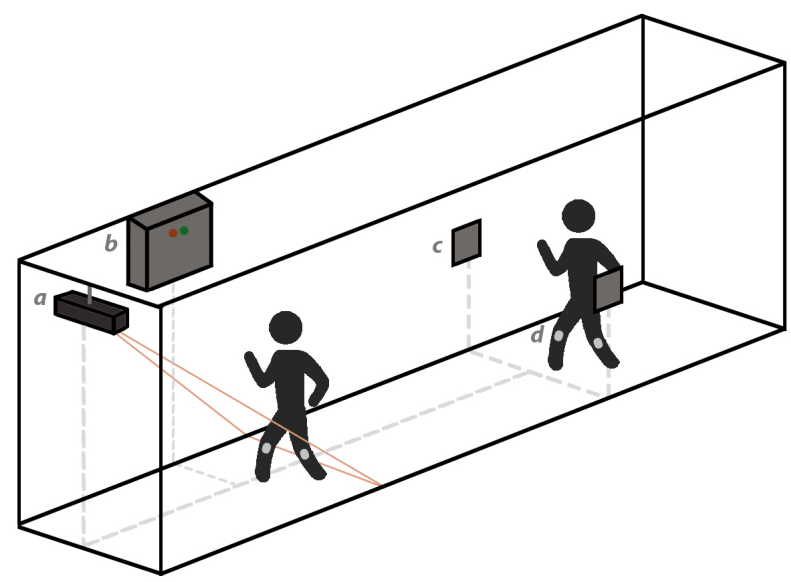

Fig. 1: The ambient monitoring system includes (a) a Kinect v2 sensor installed in a hallway, (b) two RFID antennas mounted on the hallway walls, (c) RFID tags attached to participants' clothing at knee level, (d) and a control box consisting of a laptop and an RFID reader.

\section{Related Work}

Current approaches to fall prevention in LTC settings involve some combination of baseline risk factor assessment and a multicomponent, individualized falls and injury prevention plans [9]. There are multiple concerns regarding the feasibility of these approaches in the LTC population [10]-[12]. For example, over $60 \%$ of LTC residents have dementia [13] and many have difficulty following instructions and steps required to complete clinical fall risk assessments. It is therefore desirable to develop and validate methods that could unobtrusively assess fall risk in this population.

Wearable technologies have been used in multiple studies to assess fall risk in older adults [7], [14]-[16]. While prospective fall-risk prediction via wearable sensors is potentially very useful for cognitively healthy older adults, compliance and adherence challenges limit the use of sensors in individuals with the dementia. Ambient monitoring offers an opportunity to frequently monitor gait parameters for older adults with dementia, bypassing the need for explicit adherence. There are also commercially available fall detection systems and numerous research studies on the development and evaluation of such technologies [17]. These devices and technologies are useful and potentially life saving, as they can automatically identify emergency situations and call for help. But they will not help prevent falls or fall-related injuries.

Studies have shown that people with dementia have slower gait speed, shorter stride length, greater cycle-to-cycle gait variability, and higher center of pressure variability than cognitively healthy older adults [18]-[21]. Gait and balance impairments are associated with increased risk of falling in cognitively healthy older people [21], as well as in older adults with dementia [18], [21]-[25].

In a previous analysis of the dataset used in this study, baseline gait variables measured during the first two weeks after admission could predict the number of falls experienced by an older adult with dementia during the remainder of their hospital stay [23]. In single variable analyses, the estimated lateral margin of stability, step width, and step time variability were significantly associated with number of future falls. Importantly, in multivariate analysis controlling for clinical and demographic variables, the lateral margin of stability remained associated with the number of future falls. When cox proportional hazards regression analysis was used to build a prognostic model to determine fall-free survival probabilities, gait stability (in addition to fall history) was shown to be a statistically significant predictor of time to fall in this population [26]. These studies, however, only analysed the first two weeks of gait data to estimate the number of future falls or to estimate fall-free survival probabilities. This limits the ability of developed models to dynamically update fall risk when new gait data is captured.

There are also some contradictory findings on the relationship between gait measures and falls [21]. For instance, while some studies showed that gait variability was able to discriminate between fallers and non-fallers [18], [22], others [27] found that the coefficient of variability in step time and stance time did not differentiate fallers from non-fallers. Another study [28] concluded that cadence was the only gait metric predictive of falls. These inconsistencies motivate further research in establishing features that are consistent predictors of falls, especially in high-risk populations such as individuals with dementia.

In addition, while existing studies have linked baseline gait assessments with future falls over the long-term, estimating the shortterm dynamic falling risk provides care staff with more actionable information to intervene. To improve upon static risk assessments, it is necessary to establish whether intrinsic fall risk varies over time in a measurable way, allowing for frequently updating the falling risk for each individual, e.g. based on recent changes in their gait [29]. Augmenting this information with static fall risk scores and available extrinsic risk factors (e.g. medication intake) could potentially lead to accurate dynamic fall risk assessment.

\section{Methods}

\section{A. Participants}

The participants in this study were inpatients in the Specialized Dementia Unit at the Toronto Rehabilitation Institute - an 18-bed tertiary facility which admits older patients with behavioral and psychological symptoms of dementia. There were two inclusion criteria: first, the diagnosis of major neurocognitive disorder (dementia) based on a geriatric psychiatric assessment, and second, ability to walk independently over at least 20 meters. There were no exclusion criteria. The Research Ethics Board of the University Health Network approved this study and substitute decision makers provided written informed consent for all participants. The IRB protocol number of this study is 15-9693.10, and it was approved on Dec 21, 2015. For every assessment, participants were engaged in the study provided their assent and were excluded when showing signs of dissent. Longitudinal data collection from each participant began soon after their admission (after obtaining informed consent), and continued until the end of their stay at the unit or until they were no longer able to walk independently over at least 20 meters.

\section{B. Data Collection}

Data collected from participants consists of longitudinal and baseline measures. Longitudinal measures include gait parameters from patients' walking bouts, collected via ambient monitoring, and daily medication intake, including both prescribed and as-needed (pro re nata) medication dosages. Baseline measures include assessments of gait, balance and fall risk administered at the time of admission. In addition, the dataset includes the date and time of all participants' falls that were noted during their stay at the hospital.

1) Measures of Gait: Gait measurements were collected via an ambient monitoring system (Figure 1) consisting of a depth sensor (Microsoft Kinect for Windows v2) and radio-frequency identification (RFID) to identify study participants [31]. The sensor was mounted in a hallway and captured residents' gait as they walked naturally. The RFID system consists of a reader (UHF Long Range from 


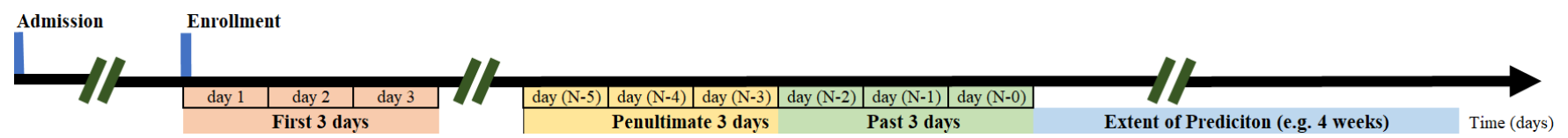

Fig. 2: Gait and medication features averaged over the past $k$ days, the penultimate $k$ days, and the first $k$ days were used to predict falls over the next few weeks. The schematic illustrates $k=3$ and the Extent of Prediction $(E o P)=4$ weeks.

TABLE I: Demographic and clinical assessment scores for study participants, summarized by faller and non-faller groups. POMA stands for the Tinetti Performance Oriented Mobility Assessment [30].

\begin{tabular}{|c|c|c|c|}
\cline { 2 - 4 } & $\begin{array}{c}\text { Total }(\text { mean } \pm \text { SD) } \\
(\mathrm{N}=54)\end{array}$ & $\begin{array}{c}\text { Non-fallers (mean } \pm \text { SD) } \\
(\mathrm{N}=34)\end{array}$ & $\begin{array}{c}\text { Fallers }(\mathrm{mean} \pm \mathrm{SD}) \\
(\mathrm{N}=20)\end{array}$ \\
\hline \hline Age (years) & $76.4 \pm 7.9$ & $75.4 \pm 7.4$ & $77.9 \pm 8.5$ \\
\hline Sex (\# men) & 30 & 19 & 11 \\
\hline Number of walks & $89.9 \pm 71.5$ & $73.6 \pm 44.5$ & $80.4 \pm 97.3$ \\
\hline Gait recording period (days) & $56.8 \pm 66.0$ & $40.6 \pm 18.9$ & $163.5 \pm 8.3$ \\
\hline Height (cm) & $163.6 \pm 14.2$ & $163.6 \pm 17.3$ & $65.9 \pm 11.2$ \\
\hline Body mass (kg) & $66.8 \pm 12.9$ & $67.6 \pm 14.1$ & $8.1 \pm 3.3$ \\
\hline POMA-balance & $9.4 \pm 3.1$ & $10.3 \pm 2.7$ & $2.9 \pm 2.6$ \\
\hline POMA-gait & $8.6 \pm 2.5$ & $9.0 \pm 2.4$ & 41.0 \\
\hline STRATIFY & $2.3 \pm 1.1$ & $2.0 \pm 0.9$ & 1.4 \\
\hline Fall history (\% previous fallers) & 29.6 & 21.9 & \\
\hline
\end{tabular}

FEIG Electronics, Duluth, Georgia, USA) and two circular polarized UHF antennas (Times-7, Wellington, New Zealand), and allows for automatically tagging participants' ID number to their gait recordings, and also (for privacy reasons) turning off the sensor when staff or nonparticipating residents are within view. The Kinect sensor tracks the human pose and motion within its field of view at the real-time [32] and previous work has established the validity of gait parameters extracted from the Kinect skeletal tracking sequences [33]. A total of 4 gait parameters were extracted from 3D skeletal tracking of each walking bout. These include: step width $(\mathrm{m})$, step time variability - quantified via the coefficient of variation (CV) of step time estimated margin of stability (eMOS), and estimated parkinsonism score - quantified via the MDS-UPDRS part III gait score. The first 3 were chosen from a longer list of available features based on a recent study which demonstrated that these 3 gait features, measured during the first two weeks of stay, were individually predictive of the number of future falls during the remaining period of hospital stay in this population [23]. Details of how these measures werre calculated from tracked skeletal sequences can be found elsewhere [23]. The fourth gait feature (UPDRS-III-gait) was estimated from tracked skeletal sequences using a spatial-temporal graph convolutional neural network (ST-GCN), via a pre-trained and previously validated model [34]. This measure was included as previous studies have linked parkinsonism and fall risk in individuals with dementia [35], [36].

2) Medications: Participants' medication intake was represented with 3 features, each of which corresponding to the daily exposure to a standardized dosage in one of the antidepressant (AD), antipsychotic (AP), and benzodiazepine (BE) categories. Using the WHO Defined Daily doses for each medication to define equivalence, all medication doses within each class were standardized to a Citalopram, Risperidone, or Lorazepam equivalent dose, and total exposure over a 24 hour period calculated.

Drug-related hypotension has been shown to be associated with falls in frail older adults [36]. Previous studies have also linked medications with central nervous system effects (including antipsychotics and antidepressants) to an increased fall risk in older adults with dementia in a dose dependent way [37]. Therefore, it was expected that including medication exposure features would result in improved fall prediction performance.
3) Baseline Assessments: Baseline assessments included in this analysis were scores of the following clinical assessments at the time of admission: the St. Thomas Risk Assessment Tool in Falling elderly inpatients (STRATIFY) [38] and the Tinetti Performance Oriented Mobility Assessment (POMA) [30] to assess gait (POMAgait) and balance (POMA-balance). Both STRATIFY and POMA scores have been previously linked to falls in older adults [39]-[41] and also specifically in older adults with dementia [23]. Despite implementation challenges that prevent regular evaluations using these clinical assessment tools [10], [12], it is often feasible to administer them once at time of admission to LTC. It was hypothesized that the baseline values of these assessments will act as a prior and may improve the predictive power of machine learning models for estimating dynamic fall risk.

4) Falls: Falls were recorded prospectively by gathering details at daily safety huddles with staff members as well as reviewing incident reports and falls documentation in charts.

For the analysis in this paper, we focused on falls which took place while standing or walking. We thus excluded any falls from chairs or beds. We also excluded any falls where there was a clear external factor leading to the fall (for example being pushed, tripping on obstacle.) Unwitnessed falls where the cause was unknown were included.

\section{Fall Risk Prediction}

A combination of longitudinal and baseline features was fed to a binary classification model trained to predict whether a person would fall over the subsequent few weeks or not. The main model presented in this work used the combination of 4 gait features, AP medication, and STRATIFY. Using a sliding window over the length of stay, the model was trained to predict falls over the subsequent 4 weeks. The effects of various other feature combinations and also the effect of changing the extent of prediction $(E o P)$ from 4 weeks to shorter or longer periods (from 1 to 11 weeks) were experimentally investigated.

For longitudinal features (gait and medication), the average values over the past $k$ days, the penultimate $k$ days, and the first $k$ days were used (Figure 2). Including features from the past $k$ days and the penultimate $k$ days allowed the model to incorporate information not only about the current gait or medication status, but also about recent changes in them in its decision making. Including features from the 
first $k$ days allows the model to also incorporate information about long-term changes in gait and medication its decision making. The effect of including each feature set (i.e. past $k$ days, penultimate $k$ days, and first $k$ days) was examined experimentally. Primary results included here are with $k=3$, but analyses with other values of $k$, ranging from 1 to 7 , are also included.

A Support Vector Machine (SVM) model with a radial basis function (RBF) kernel was used as a binary classifier to distinguish participants who fell once or more during the subsequent four weeks vs. those who did not. Other classification models (SVM with a linear kernel, logistic regression, and random forest) yielded similar (but slightly worse) results in preliminary experiments and, for brevity, are not presented. The kernel coefficient was set to (1 / \#features). Positive (falls) and negative (non-fall) cases were weighted proportionally to the inverse of their respective frequencies.

For each participant, a fall risk prediction is made on every day on where there was at least one gait recording on the preceding $k$ days. The output, via Platt scaling, is a number between 0 to 1 , which can be interpreted as the participant's falling risk (probability) in next four weeks.

\section{Train, Test, and Validation Split}

In nested cross-validation, data was split into training, validation, and test sets so that test performance could be reported on the entire dataset while the regularization hyperparameter was finetuned over the validation set. The split between the $\{$ Test $\}$ and \{Train,Validation\} sets was leave-one-subject-out, ensuring that data from each test participant did not appear in either the training or validation sets. In each data split, the features in all three sets were normalized according to the range of values in the training set.

\section{E. Performance Evaluation}

To investigate the effect of $k$, the effect of the fall prediction extent, and the contribution of each set of features to the overall performance, multiple models were trained using various combinations of features and hyperparameters. The primary metric used to compare model performance was the area under the receiver operating characteristic curve (AUROC), but the area under the precision-recall curve (average precision), as well as precision, recall, specificity, and the F1-score at the default threshold value (0.5) are also reported.

\section{Results And Discussion}

During $\sim 22$ months of data collection, 64 individuals with dementia were recruited to participate in this study. One person withdrew their consent shortly after entry and 4 others were withdrawn due to changes in health status such that they could no longer walk independently over at least 20 meters before any gait recordings were completed. Of the remaining 59 participants, 5 with no recorded walks were excluded and the the remaining 54 participants were included in the analysis.

There were a total of 81 falls during the period of observation. Among these, 19 were caused by external factors (being pushed etc.) and 8 occurred while transferring from a sitting or lying position, of which 1 overlapped with the 19 caused by external factors. As such, there were a total of $(81-19-8+1=) 55$ falls included in the analysis. Twenty (20) of the 54 participants fell at least once since their first gait-recorded walk and up to 4 weeks after their latest gait-recorded walk. The gait recording period (from the first ambient gait recording until the last) among all 54 participants was 56.8 days on average. A total of 4851 walking bouts were collected over the participants' length of stay. The recorded number of walking bouts per patient varied from a minimum of 11 to a maximum of 323. Table I summarizes the demographic information of study participants. Finally, a total of 17,114 medication administration events were collected over the participants' length of stay

The fall risk prediction model achieved an AUROC of $75.8 \%$, recall (sensitivity) of $75.5 \%$, specificity of $71.9 \%$, and precision (positive predictive value) of $41.6 \%$, amounting to an F1-score of of $53.7 \%$. This model was based on 4 longitudinal gait features (step width, CV of step time, eMOS, UPDRS-III-gait), daily standardized dosage of AP medication, and baseline STRATIFY scores. The values of $k$ and $E o P$ were 3 days and 4 weeks, respectively. For longitudinal features, the average values over the past 3 days, the penultimate 3 days, and the first 3 days were used. This amounted to using a total of $(4+1) \times 3+1=16$ features.

Table II shows the effect of varying $k$ from 1 to 7 days. The best AUROC was achieved at $k=3$, but changes in the AUROC are relatively small when different values of $k$ are used. Specifically, AUROC $=75.8$ at $k=3$ and AUROC $=74.2$ at $k=4$, and all values of $k$ ranging from 1 to 7 resulted in AUROC values over 72 .

Table III shows the effect of various combinations of longitudinal data from the past 3 days (PAST), the penultimate 3 days (PENULTIMATE), and the first 3 days (FIRST). The best AUROC is obtained when data from the past, penultimate, and first three days are used. Including only the past and the first groups degrades performance by only a small amount (AUROC 74.1 vs. 75.8). In contrast, removing the first group and only using the past and penultimate groups greatly degrades performance to an AUROC of 68.6. This implies that changes in gait over multiple weeks (from the first until the final week) are better predictors of fall risk than the most recent changes in gait (over the past 6 days).

Table IV shows the model's performance when the extent of prediction ranges from 1 to 11 weeks. In all the experiments, replacing STRATIFY with POMA scores resulted in a poorer performance. Similarly, including both STRATIFY and POMA scores did not improve performance and in some cases even slightly lowered performance, perhaps due to overfitting. For brevity, only results with STRATIFY are reported here.

From the results presented in Table IV, it can be seen that best AUROC of 78.3 is achieved with $E o P=6$ weeks and that AUROC remained above 75 for $E o P$ ranging from 4 to 7 weeks. Performance dropped below an AUROC of 70 when $E o P$ is set to 1 or 2 weeks. While the best AUROC is achieved at $E o P=6$ weeks, the model chosen for further analysis in this study was with $E o P=4$ weeks, i.e. the smallest $E o P$ with AUROC greater than 75.0. The difference in AUROC when changing EoP from 6 to 4 weeks is relatively small (75.8 vs. 78.3), and a shorter term fall risk score provides with clinicians and care staff with more actionable knowledge and better chance to intervene.

Depending on the EoP, only $8-21 \%$ of the data points belong to the positive class (falls). The second column of Table IV shows the percentage of positive cases for each EoP. Without balancing class weights, the AUROC of the model with $E o P=4$ drops from $75.8 \%$ to $62.3 \%$. This drop highlights the importance of applying class weights to put more emphasis on the minority class. In addition, the large drop in AUROC when $E o P \leqslant 2$ corresponds with a drop in the percentage of true positives to below $15 \%$, potentially because of the reduction in the diversity of positive class data points which inhibits the models' generalizability.

Table $\mathrm{V}$ shows the effect of removing each feature group on fall prediction performance. Figure 3 plots the ROC curve for the best model with $E o P=4$ (top row in Table V), the model with the same feature set excluding AP ( $2^{\text {nd }}$ row in Table V), the model based only 
TABLE II: Effect of $k$ on fall prediction performance.

\begin{tabular}{|c|c|c|c|c|c|c|c|}
\hline Days & Precision (\%) & Recall (\%) & F1Score (\%) & Accuracy (\%) & Specificity (\%) & Avg Precision (\%) & AUROC \\
\hline \hline 1 & 38.4 & 75.9 & 51.0 & 68.8 & 66.9 & 37.0 \\
\hline 2 & 39.8 & 73.5 & 51.6 & 70.2 & 69.3 & 71.5 \\
\hline 3 & 41.6 & 75.5 & 53.7 & 72.6 & 71.9 & 73.1 \\
\hline 4 & 40.5 & 74.3 & 52.4 & 71.9 & 71.2 & 71.9 & 75.8 \\
\hline 5 & 39.0 & 66.3 & 49.1 & 71.4 & 72.8 & 74.0 \\
\hline 6 & 39.1 & 68.1 & 49.7 & 71.3 & 72.1 & 73.1 \\
\hline 7 & 38.1 & 65.3 & 48.1 & 70.7 & 72.2 & 38.3 & 73.3 \\
\hline
\end{tabular}

$E o P=4$, longitudinal features from the PAST \& PENULTIMATE \& FIRST $k$ days, Features = Gait (Step Width, CV Step Time, eMOS, UPDRS-III-gait, Medications (AP), and STRATIFY.

TABLE III: Effect of longitudinal feature group on fall prediction performance.

\begin{tabular}{|c|c|c|c|c|c|c|c|}
\hline Longitudinal Feature Group & Precision $(\%)$ & Recall (\%) & F1Score $(\%)$ & Accuracy $(\%)$ & Specificity (\%) & Avg Precision (\%) & AUROC \\
\hline PAST + PENULTIMATE + FIRST & 41.6 & 75.5 & 53.7 & 72.6 & 71.9 & 42.9 & 75.8 \\
\hline PAST + FIRST & 41.1 & 78.8 & 54.1 & 71.8 & 70.0 & 39.6 & 74.1 \\
\hline PAST + PENULTIMATE & 37.9 & 55.6 & 45.0 & 71.5 & 75.7 & 37.9 & 68.6 \\
\hline FIRST & 23.4 & 61.0 & 33.9 & 50.0 & 47.0 & 29.8 & 57.4 \\
\hline
\end{tabular}

$E O P=4, k=3$, Features = Gait (Step Width, CV Step Time, eMOS, UPDRS-III-gait), Medications (AP), and STRATIFY.

TABLE IV: Effect of EoP (extent of prediction) on fall prediction performance.

\begin{tabular}{|c|c|c|c|c|c|c|c|c|}
\hline$E o P$ (weeks) & Positive Cases (\%) & Precision (\%) & Recall (\%) & F1Score (\%) & Accuracy (\%) & Specificity (\%) & Avg Precision (\%) & AUROC \\
\hline$\overline{1}$ & 9.0 & 17.8 & 71.8 & 28.6 & $\overline{667.7}$ & 67.3 & 18.4 & $\overline{688.7}$ \\
\hline 2 & 13.2 & 24.0 & 68.9 & 35.6 & 67.1 & 66.9 & 21.9 & 66.2 \\
\hline 3 & 17.1 & 32.7 & 75.6 & 45.7 & 69.1 & 67.8 & 34.4 & 72.6 \\
\hline 4 & 20.6 & 41.6 & 75.5 & 53.7 & 72.6 & 71.9 & 42.9 & 75.8 \\
\hline 5 & 22.6 & 46.2 & 75.7 & 57.4 & 74.6 & 74.3 & 50.6 & 78.1 \\
\hline 6 & 23.7 & 48.7 & 76.5 & 59.5 & 75.3 & 75.0 & 52.6 & 78.3 \\
\hline 7 & 24.8 & 51.1 & 75.0 & 60.8 & 76.0 & 76.4 & 53.2 & 78.0 \\
\hline 8 & 25.9 & 49.8 & 70.0 & 58.2 & 73.9 & 75.3 & 52.3 & 74.8 \\
\hline 9 & 27.0 & 50.6 & 67.7 & 57.9 & 73.4 & 75.5 & 52.5 & 73.5 \\
\hline 10 & 27.8 & 47.9 & 66.5 & 55.6 & 70.5 & 72.1 & 49.9 & 71.3 \\
\hline 11 & 28.3 & 47.5 & 65.5 & 55.1 & 69.7 & 71.4 & 50.1 & 71.2 \\
\hline
\end{tabular}
Medications (AP), and STRATIFY.

on gait features $\left(7^{\text {th }}\right.$ row in Table $\left.\mathrm{V}\right)$, and the model based only on STRATIFY (last row in Table V).

Results in Table $\mathrm{V}$ show that the weakest classifier of all feature combinations, with an AUROC of 50.4, understandably belongs to the model solely trained on the static STRATIFY score administered at the time of admission. This model performs virtually the same as chance predictions, for which the AUROC would be 50.0. As presented in Table $\mathrm{V}$, adding longitudinal feature groups (gait or medication) improved the classifiers' performance and the highest AUROC of 75.8 is achieved when Gait, AP, and STRATIFY are used. Removing antipsychotic medication (AP) information only slightly degrades model performance to an AUROC of 74.2 ( $2^{\text {nd }}$ row in Table V). Removing Gait features results in a drop to $\mathrm{AUROC}=70.5$ when only STRATIFY and AP features are used ( $3^{\text {rd }}$ row in Table V). Removing STRATIFY and only using the longitudinal features results in a larger performance drop to an AUROC of $67.9\left(5^{\text {th }}\right.$ row in Table V). That is, while STRATIFY alone is not a good predictor of short-term falls, it serves as an important prior augmenting the longitudinal features.

Finally, Table VI shows the effect of using longitudinal features from other medication groups (BE and $\mathrm{AD}$ ) or the combination of all three medication groups (AP, BE, and AD). Including all three types of medications in the feature set resulted in a large drop in performance $($ AUROC $=62.3$ when all 3 medication groups are used vs. 75.8 when only AP medications are fed to the predictive model). This is likely due to overfitting when all three medication types are used. Replacing AP with either $\mathrm{BE}$ or $\mathrm{AD}$ information also resulted in a lower performance, with an AUROC of 64.6 and

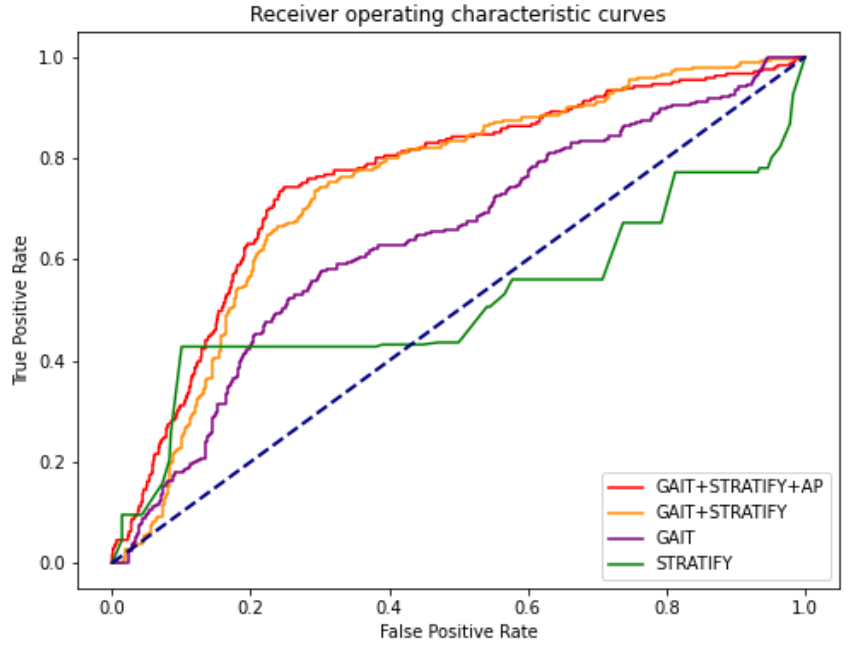

Fig. 3: ROC curves for four feature sets.

Gait $=\{$ Step Width, CV Step Time, eMOS, UPDRS $\}$

57.0 respectively. Individually, $\mathrm{AD}$ and $\mathrm{BE}$ features performed worse than chance prediction $(A U R O C<50.0)$ and $A P$ alone resulted in an AUROC of 53.5.

As the fall risk assessment model is being trained and evaluated towards the development and deployment of an automated alert system for a fall prevention program, it is important to be mindful of potential disparate impact. It is, therefore, important to investigate 
TABLE V: Effect of feature set on fall prediction performance.

\begin{tabular}{|c|c|c|c|c|c|c|c|}
\hline Features & Precision (\%) & Recall (\%) & F1Score (\%) & Accuracy (\%) & Specificity (\%) & Avg Precision (\%) & AUROC \\
\hline \hline Gait + STRATIFY + AP & 41.6 & 75.5 & 53.7 & 72.6 & 71.9 & 42.9 & 75.8 \\
\hline Gait + STRATIFY & 43.4 & 63.9 & 51.7 & 74.9 & 77.8 & 35.9 \\
\hline STRATIFY + AP & 40.2 & 64.7 & 49.6 & 72.4 & 74.4 & 35.3 \\
\hline Gait [excluding UPDRS] + STRATIFY & 36.8 & 55.2 & 44.2 & 70.7 & 74.8 & 70.5 \\
\hline Gait + AP & 32.5 & 73.0 & 45.0 & 62.5 & 59.7 & 31.9 & 70.4 \\
\hline UPDRS + STRATIFY & 35.8 & 58.9 & 44.5 & 69.1 & 71.9 & 29.4 & 67.9 \\
\hline Gait & 31.5 & 66.0 & 42.7 & 62.8 & 61.9 & 32.6 \\
\hline Gait [excluding UPDRS] & 29.5 & 58.1 & 39.1 & 62.0 & 63.0 & 27.4 \\
\hline UPDRS & 31.9 & 75.9 & 45.0 & 60.9 & 57.0 & 23.0 \\
\hline STRATIFY & 53.1 & 42.7 & 47.4 & 80.0 & 90.0 & 57.6 \\
\hline
\end{tabular}

TABLE VI: Effect of medication features on fall prediction performance.

\begin{tabular}{|c|c|c|c|c|c|c|c|}
\hline Features & Precision (\%) & Recall (\%) & F1Score $(\%)$ & Accuracy $(\%)$ & Specificity (\%) & Avg Precision (\%) & AUROC \\
\hline 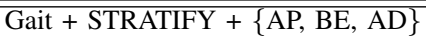 & 31.0 & $\overline{45.6}$ & 36.9 & $\overline{67.2}$ & $\overline{73.0}$ & 31.0 & $\overline{62.3}$ \\
\hline Gait + STRATIFY + AP & 41.6 & 75.5 & 53.7 & 72.6 & 71.9 & 42.9 & 75.8 \\
\hline Gait + STRATIFY + BE & 27.9 & 68.5 & 39.7 & 56.2 & 53.0 & 29.0 & 64.6 \\
\hline Gait + STRATIFY + AD & 41.7 & 41.9 & 41.8 & 75.5 & 84.4 & 35.3 & 57.0 \\
\hline$\{\mathrm{AP}, \mathrm{BE}, \mathrm{AD}\}$ & 28.6 & 39.8 & 33.3 & 66.4 & 73.5 & 24.6 & 54.1 \\
\hline $\mathrm{AP}$ & 26.2 & 87.1 & 40.3 & 45.7 & 34.7 & 20.7 & 53.5 \\
\hline $\mathrm{BE}$ & 32.3 & 30.3 & 31.3 & 72.0 & 83.1 & 24.9 & 44.3 \\
\hline $\mathrm{AD}$ & 21.8 & 73.0 & 33.6 & 39.2 & 30.2 & 16.2 & 36.9 \\
\hline
\end{tabular}

$E o P=4, k=3$, longitudinal features from the PAST \& PENULTIMATE \& FIRST $k$ days, Gait $=\{$ Step Width, CV Step Time, eMOS, UPDRS-III-gait $\}$.

TABLE VII: Model performance stratified based on sex.

\begin{tabular}{|c|c|c|c|c|c|c|c|c|}
\hline Sex & Positive Cases (\%) & Precision (\%) & Recall (\%) & F1Score (\%) & Accuracy (\%) & Specificity (\%) & Avg Precision (\%) & AUROC \\
\hline \hline Female (N=24) & 18.5 & 50.0 & 74.4 & 59.8 & 72.6 & 75.8 & 42.8 & 75.8 \\
\hline Male (N=30) & 24.5 & 36.1 & 76.6 & 49.1 & 72.6 & 69.3 & 42.8 & 75.8 \\
\hline
\end{tabular}

$E O P=4, k=3$, longitudinal features from the PAST \& PENULTIMATE \& FIRST $k$ days, Features = Gait (Step Width, CV Step Time, eMOS, UPDRS-III-gait), Medications (AP), and STRATIFY.

model performance stratified based on population groups, e.g. based on sex or ethnicity. While ethnicity/race information is not available for the study participants, it is possible to break performance down based on sex. Among the 30 male participants, $11(36.7 \%)$ were fallers. Among the 24 female participants, 9 (37.5\%) were fallers. Table VII presents the performance of the model separately for men and women in the cohort. As Table VII shows, the model obtains identical AUROC and average precision for both groups.

This study presented important findings and demonstrated the possibility of dynamic fall risk assessment in older adults with dementia via ambient monitoring. While the findings are important and novel, there are also several limitations with the current study. First, while the number of ambient gait assessments in the study is substantial (4851), the number of participants is limited to 54, thus limiting the variability in the dataset. Second, while the average number of walks per participant was relatively high (89.9, see Table I), the range varied significantly, from as low as 11 to as high as 323 . This further limits the variability captured in the dataset as individuals with more recorded walks and a longer length of stay are over-represented. Third, most participants had a short length of stay. The setting where data were collected was a specialized dementia unit which admits older adults with behavioural and psychological symptoms of dementia. The average length of stay in this unit is significantly shorter than that of LTC facilities (2-3 months vs. years). Fourth, because the unit typically admits individuals with severe behavioural and psychological symptoms who have a higher risk of falling, the rate of falls in this unit is higher than a typical LTC facility. As a result, it is important to externally validate these findings on longerterm longitudinal data collected in LTC facilities where the fall rate is expected to be lower.

\section{CONCLUSIONS AND FUtURE WORK}

This study is the first to present a machine learning model capable of dynamically estimating short-term fall risk for older adults with dementia. The model used baseline fall risk scores, gait measurements from baseline and in the past 6 days, and daily dose of antipsychotic medication in the past 6 days. The sensitivity and specificity of the model (75.5 and 71.2 for predicting falls over the next 4 weeks, and 76.5 and 75.0 for predicting falls over the next 6 weeks) are close to clinically acceptable levels, motivating further research to improve and prepare the technology for deployment.

Ongoing work includes longitudinal data collection from multiple ambient monitoring systems in other long-term care facilities to externally validate the developed machine learning models on data collected at other sites. Future work will involve deploying the dynamic fall risk assessment technology in LTC facilities to alert the care staff when a large increase in probability of a fall is detected for a resident, and to investigate the effect of this technology on reducing falls and fall related injuries in older adults with dementia.

\section{REFERENCES}

[1] Dallas P Seitz, Sudeep S Gill, Andrea Gruneir, Peter C Austin, Geoffrey M Anderson, Chaim M Bell, and Paula A Rochon. Effects of dementia on postoperative outcomes of older adults with hip fractures: a population-based study. Journal of the American Medical Directors Association, 15(5):334-341, 2014.

[2] Pieter TM van Dijk, Oda GRM Meulenberg, Herbert J Van de Sande, and J Dik F Habbema. Falls in dementia patients. The Gerontologist, 33(2):200-204, 1993.

[3] Mary E Tinetti, Mark Speechley, and Sandra F Ginter. Risk factors for falls among elderly persons living in the community. New England journal of medicine, 319(26):1701-1707, 1988.

[4] FE Shaw. Prevention of falls in older people with dementia. Journal of neural transmission, 114(10):1259-1264, 2007. 
[5] Gisela Büchele, Clemens Becker, Ian D Cameron, Hans-Helmut König, Stephen Robinovitch, and Kilian Rapp. Predictors of serious consequences of falls in residential aged care: analysis of more than 70,000 falls from residents of bavarian nursing homes. Journal of the American Medical Directors Association, 15(8):559-563, 2014.

[6] Jim George, Susannah Long, and Charles Vincent. How can we keep patients with dementia safe in our acute hospitals? a review of challenges and solutions. Journal of the Royal Society of Medicine, 106(9):355-361, 2013.

[7] Roman Schniepp, Anna Huppert, Julian Decker, Fabian Schenkel, Cornelia Schlick, Atal Rasoul, Marianne Dieterich, Thomas Brandt, Klaus Jahn, and Max Wuehr. Fall prediction in neurological gait disorders: differential contributions from clinical assessment, gait analysis, and daily-life mobility monitoring. Journal of neurology, pages 1-14, 2021.

[8] Robert L Berg, Joseph S Cassells, et al. Falls in older persons: risk factors and prevention. In The second fifty years: Promoting health and preventing disability. National Academies Press (US), 1992.

[9] Registered Nurses' Association of Ontario. Preventing falls and reducing injury from falls, 2017.

[10] Carolyn S Sterke, Sawadi L Huisman, Ed F van Beeck, Caspar WN Looman, and Tischa JM van der Cammen. Is the tinetti performance oriented mobility assessment (poma) a feasible and valid predictor of short-term fall risk in nursing home residents with dementia? International psychogeriatrics, 22(2):254-263, 2010.

[11] Mary E Tinetti, Catherine Gordon, Ellen Sogolow, Pauline Lapin, and Elizabeth H Bradley. Fall-risk evaluation and management: challenges in adopting geriatric care practices. The Gerontologist, 46(6):717-725, 2006.

[12] Karen Van Ooteghem, Kristin Musselman, David Gold, Meghan N Marcil, Ron Keren, Maria Carmela Tartaglia, Alastair J Flint, and Andrea Iaboni. Evaluating mobility in advanced dementia: a scoping review and feasibility analysis. The Gerontologist, 59(6):e683-e696, 2019.

[13] Pouria Mashouri, Babak Taati, Hannah Quirt, and Andrea Iaboni. Quality indicators as predictors of future inspection performance in ontario nursing homes. Journal of the American Medical Directors Association, 21(6):793-798, 2020.

[14] Luis Montesinos, Rossana Castaldo, and Leandro Pecchia. Wearable inertial sensors for fall risk assessment and prediction in older adults: a systematic review and meta-analysis. IEEE transactions on neural systems and rehabilitation engineering, 26(3):573-582, 2018.

[15] Jennifer Howcroft, Jonathan Kofman, and Edward D Lemaire. Prospective fall-risk prediction models for older adults based on wearable sensors. IEEE transactions on neural systems and rehabilitation engineering, 25(10):1812-1820, 2017.

[16] Patricia Bet, Paula C Castro, and Moacir A Ponti. Fall detection and fall risk assessment in older person using wearable sensors: A systematic review. International journal of medical informatics, 130:103946, 2019.

[17] Shehroz S Khan and Jesse Hoey. Review of fall detection techniques: A data availability perspective. Medical engineering \& physics, 39:12-22, 2017.

[18] Ellen L McGough, Rebecca G Logsdon, Valerie E Kelly, and Linda Teri. Functional mobility limitations and falls in assisted living residents with dementia: physical performance assessment and quantitative gait analysis. Journal of geriatric physical therapy, 36(2):78-86, 2013.

[19] Joanne E Wittwer, Kate E Webster, and Hylton B Menz. A longitudinal study of measures of walking in people with alzheimer's disease. Gait \& Posture, 32(1):113-117, 2010.

[20] Pamela L Sheridan, Judi Solomont, Neil Kowall, and Jeffrey M Hausdorff. Influence of executive function on locomotor function: divided attention increases gait variability in alzheimer's disease. Journal of the American Geriatrics Society, 51(11):1633-1637, 2003.

[21] Elham Dolatabadi, Karen Van Ooteghem, Babak Taati, and Andrea Iaboni. Quantitative mobility assessment for fall risk prediction in dementia: a systematic review. Dementia and geriatric cognitive disorders, 45(5-6):353-367, 2018.

[22] Debra Morgan, Melanie Funk, Margaret Crossley, Jenny Basran, Andrew Kirk, and Vanina Dal Bello-Haas. The potential of gait analysis to contribute to differential diagnosis of early stage dementia: current research and future directions. Canadian Journal on Aging/La Revue canadienne du vieillissement, 26(1):19-32, 2007.

[23] Sina Mehdizadeh, Elham Dolatabadi, Kimberley-Dale Ng, Avril Mansfield, Alastair Flint, Babak Taati, and Andrea Iaboni. Vision-based assessment of gait features associated with falls in people with dementia. The Journals of Gerontology: Series A, 75(6):1148-1153, 2020.

[24] Kimberley-Dale Ng, Sina Mehdizadeh, Andrea Iaboni, Avril Mansfield, Alastair Flint, and Babak Taati. Measuring gait variables using computer vision to assess mobility and fall risk in older adults with dementia. IEEE
Journal of Translational Engineering in Health and Medicine, 8:1-9, 2020.

[25] Risk factors for falls in older people with cognitive impairment living in the community: systematic review and meta-analysis. Ageing Research Reviews, page 101452, 2021.

[26] Sina Mehdizadeh, Andrea Sabo, Kimberley-Dale Ng, Avril Mansfield, Alastair J Flint, Babak Taati, and Andrea Iaboni. Predicting short-term risk of falls in a high-risk group with dementia. Journal of the American Medical Directors Association, 22(3):689-695, 2021.

[27] Jennifer S Brach, Jaime E Berlin, Jessie M VanSwearingen, Anne B Newman, and Stephanie A Studenski. Too much or too little step width variability is associated with a fall history in older persons who walk at or near normal gait speed. Journal of neuroengineering and rehabilitation, 2(1):1-8, 2005.

[28] Andrea Merlo, Damiano Zemp, Enrica Zanda, Sabrina Rocchi, Fabiano Meroni, Mauro Tettamanti, Angela Recchia, Ugo Lucca, and Pierluigi Quadri. Postural stability and history of falls in cognitively able older adults: The canton ticino study. Gait \& posture, 36(4):662-666, 2012.

[29] Jochen Klenk, Clemens Becker, Pierpaolo Palumbo, Lars Schwickert, Kilan Rapp, Jorunn L Helbostad, Chris Todd, Stephen R Lord, and Ngaire Kerse. Conceptualizing a dynamic fall risk model including intrinsic risks and exposures. Journal of the American Medical Directors Association, 18(11):921-927, 2017.

[30] Mary E Tinetti. Performance-oriented assessment of mobility problems in elderly patients. Journal of the American Geriatrics Society, 34(2):119-126, 1986.

[31] Elham Dolatabadi, Ying Xuan Zhi, Alastair J Flint, Avril Mansfield, Andrea Iaboni, and Babak Taati. The feasibility of a vision-based sensor for longitudinal monitoring of mobility in older adults with dementia. Archives of gerontology and geriatrics, 82:200-206, 2019.

[32] Jamie Shotton, Andrew Fitzgibbon, Mat Cook, Toby Sharp, Mark Finocchio, Richard Moore, Alex Kipman, and Andrew Blake. Real-time human pose recognition in parts from single depth images. In CVPR 2011, pages 1297-1304. Ieee, 2011.

[33] Elham Dolatabadi, Babak Taati, and Alex Mihailidis. Concurrent validity of the microsoft kinect for windows v2 for measuring spatiotemporal gait parameters. Medical engineering \& physics, 38(9):952-958, 2016.

[34] Andrea Sabo, Sina Mehdizadeh, Andrea Iaboni, and Babak Taati. Estimating parkinsonism severity in natural gait videos of older adults with dementia. arXiv preprint arXiv:2105.03464, 2021.

[35] Clive G Ballard, Fiona Shaw, Kathleen Lowery, Ian McKeith, and Roseanne Kenny. The prevalence, assessment and associations of falls in dementia with lewy bodies and alzheimer's disease. Dementia and geriatric cognitive disorders, 10(2):97-103, 1999.

[36] Lewis A Lipsitz, Palmi V Jonsson, Margaret M Kelley, and Julia S Koestner. Causes and correlates of recurrent falls in ambulatory frail elderly. Journal of gerontology, 46(4):M114-M122, 1991.

[37] Eresha Fernando, Michelle Fraser, Jane Hendriksen, Corey H Kim, and Susan W Muir-Hunter. Risk factors associated with falls in older adults with dementia: a systematic review. Physiotherapy Canada, 69(2):161170,2017

[38] David Oliver, M Britton, P Seed, FC Martin, and AH Hopper. Development and evaluation of evidence based risk assessment tool (stratify) to predict which elderly inpatients will fall: case-control and cohort studies. Bmj, 315(7115):1049-1053, 1997.

[39] Koen Milisen, Nele Staelens, René Schwendimann, Leen De Paepe, Jeroen Verhaeghe, Tom Braes, Steven Boonen, Walter Pelemans, Reto W Kressig, and Eddy Dejaeger. Fall prediction in inpatients by bedside nurses using the st. thomas's risk assessment tool in falling elderly inpatients (stratify) instrument: a multicenter study. Journal of the American Geriatrics Society, 55(5):725-733, 2007.

[40] Fulvio Lauretani, Andrea Ticinesi, Luciano Gionti, Beatrice Prati, Antonio Nouvenne, Claudio Tana, Tiziana Meschi, and Marcello Maggio. Short-physical performance battery (sppb) score is associated with falls in older outpatients. Aging clinical and experimental research, 31(10):1435-1442, 2019.

[41] Marjan J Faber, Ruud J Bosscher, and Piet CW van Wieringen. Clinimetric properties of the performance-oriented mobility assessment. Physical therapy, 86(7):944-954, 2006. 